\title{
Optimal electrode setting method in brain machine interface
}

\author{
yanli yuan ${ }^{1}$, Bin LV ${ }^{1}$, Yuzhi Sun ${ }^{2}$, and Tianmin Guan ${ }^{1}$ \\ ${ }^{1}$ Dalian Jiaotong University \\ ${ }^{2}$ Oregon State University
}

May 5, 2020

\begin{abstract}
The process of intelligent interaction through brain machine interface requires quick and accurate extraction of Electroencephalogram (EEG) signals. However, the accuracy of signal classification varies with the signal extraction location. Is there a universal rule to follow to determine the optimal extraction location? This paper investigates the possibility of a universal rule to determine optimal extraction location through Welch, Support Vector Machine and Euclidean distance algorithms. The motor imagery EEG signals of 40 subjects were extracted and the classification correct rates of brain electrode signals in different positions were analyzed using Welch and Support Vector Machine algorithms. Then the electrodes were sorted according to the correct rate, and finally three pairs of electrodes with the highest correct rate were obtained. For comparison, this paper proposed another method of searching for the optimal electrodes, namely the combination of Welch and Euclidean distance algorithms. Ultimately, a similar conclusion was drawn from the above two methods: T3/T4, F3/F4 and C3/C4 electrodes usually have high classification accuracy. This result is helpful for quick customization of optimal brain electrode placement.
\end{abstract}

\section{Hosted file}

Optimal_electrode_setting_method_in_brain_machine_interface.doc available at https: //authorea.com/users/293047/articles/420931-optimal-electrode-setting-method-in-brainmachine-interface 


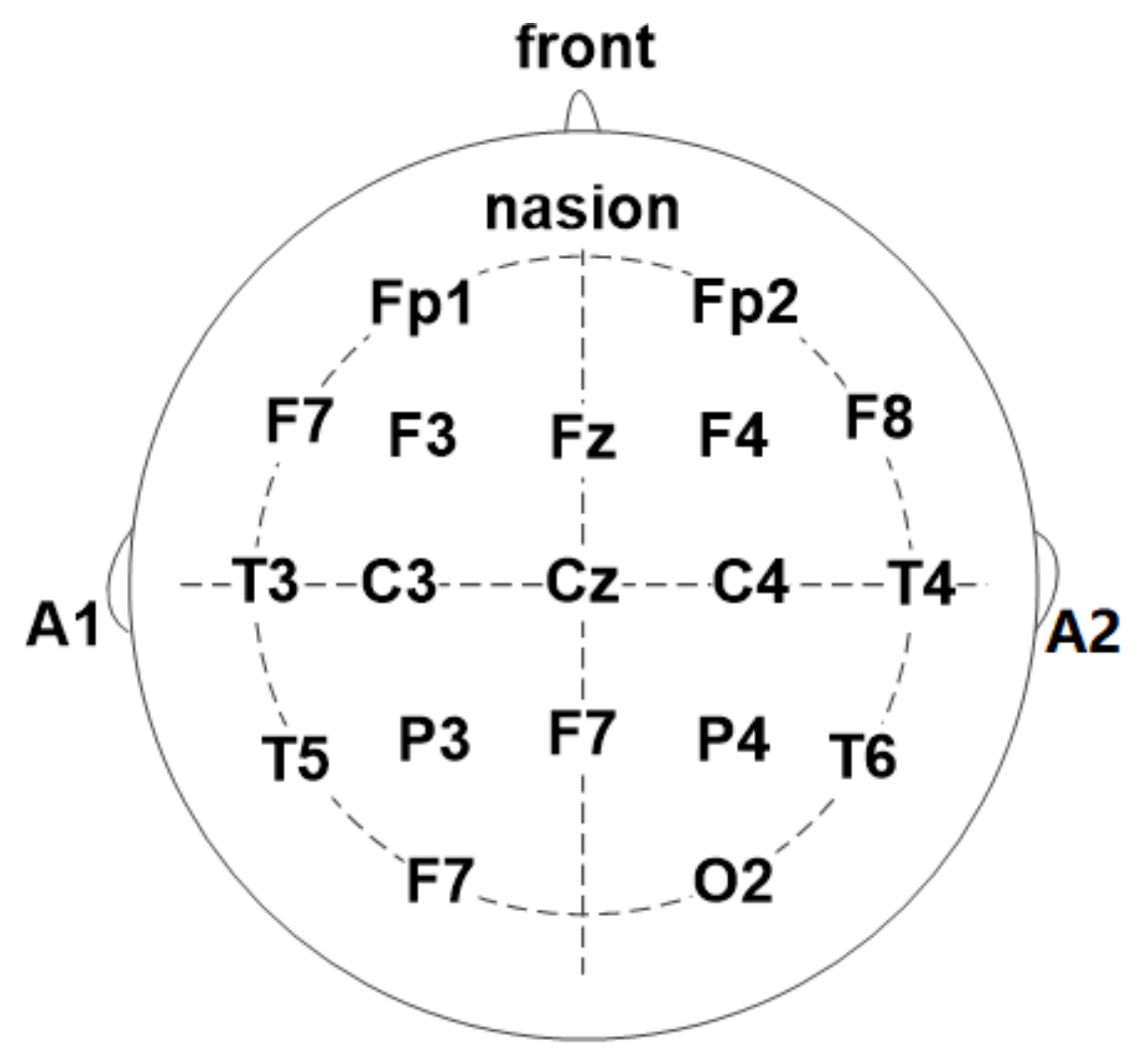

FIGURE 1 Electrode position of the system

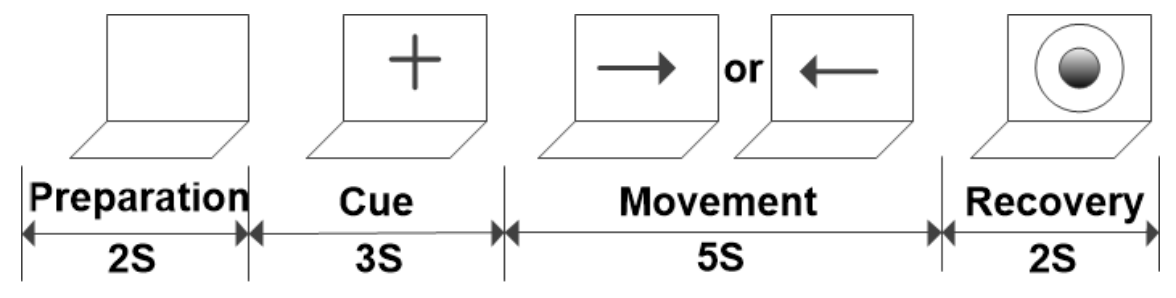

FIGURE 2 The experimental procedure 


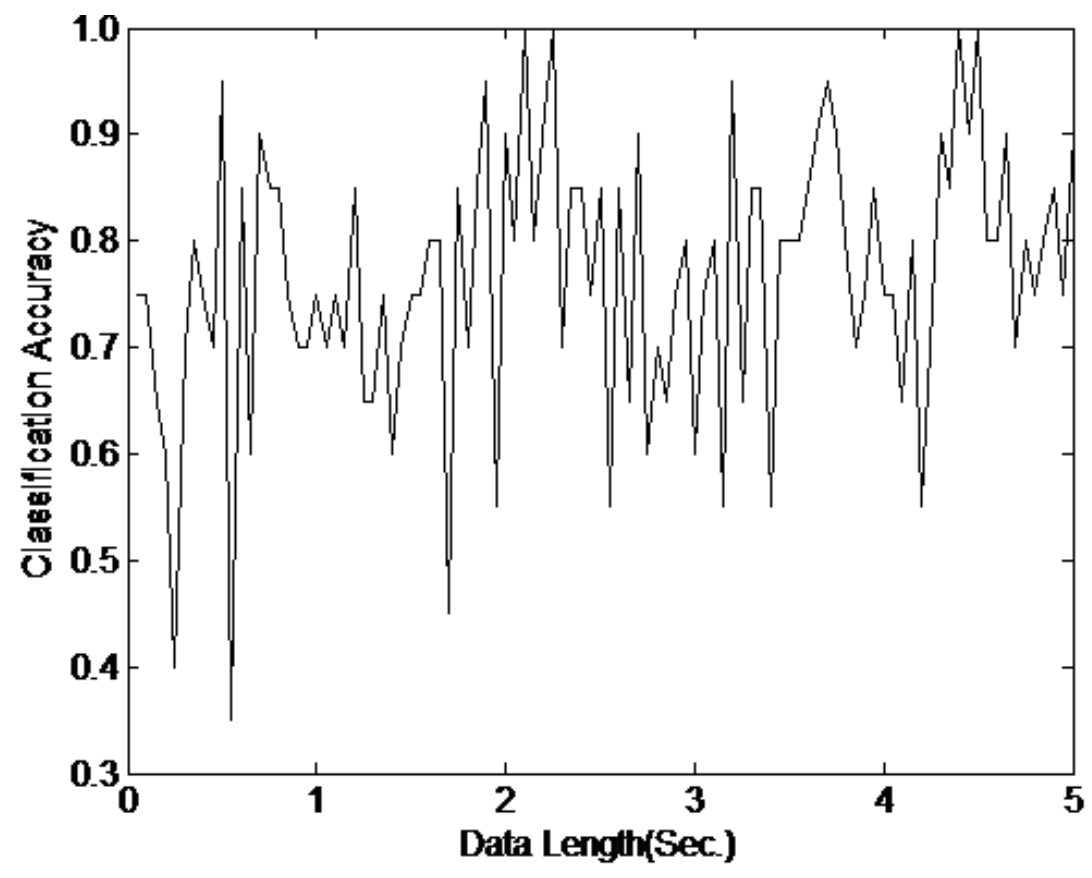

FIGURE 3 LQ's classification accuracy in C3 C4

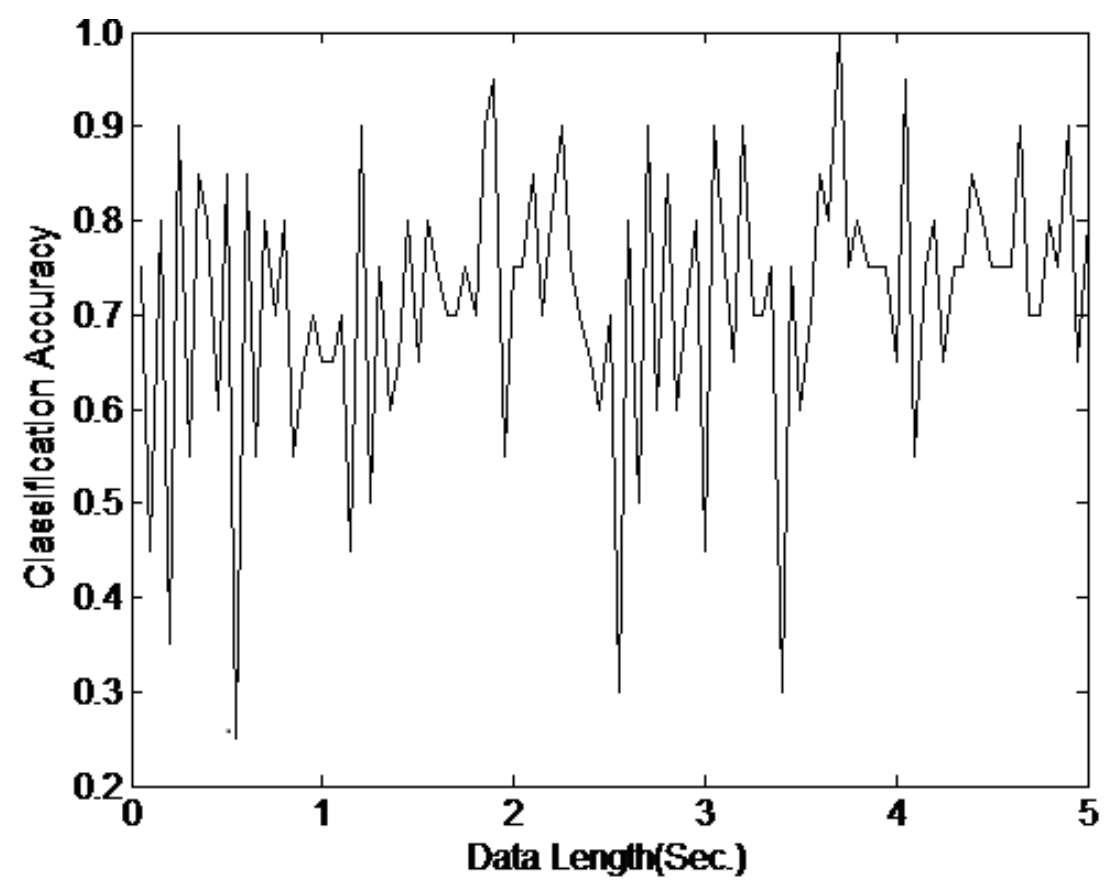

FIGURE 4 LQ's classification accuracy in F3 F4 